Arq. Bras. Med. Vet. Zootec., v.68, n.4, p.887-893, 2016

\title{
Deformação miocárdica radial por meio do speckle tracking bidimensional em suínos com hipertensão pulmonar induzida e tratados com angiotensina-(1-7)
}

[Evaluation of myocardial radial deformation by bidimensional speckle tracking in pigs with pulmonary hypertension treated with angiotensin- (1-7)]

M.R. Coelho, L.E.D. Oliveira, R.A.L. Muzzi, G. Oberlender, M.S. Varaschin, R.C. Costa, L.A.L. Muzzi

Universidade Federal de Lavras - Lavras, MG

\section{RESUMO}

A hipertensão arterial pulmonar (HAP) é uma doença complexa, caracterizada por disfunção endotelial, que resulta em remodelamento vascular pulmonar e elevação da pressão arterial pulmonar, com consequente insuficiência cardíaca direita. O speckle tracking bidimensional (2D-STE) é uma das mais recentes ferramentas da ecocardiografia, o qual tem sido empregado para avaliação mais precoce da função ventricular e do efeito da HAP sobre a função dos ventrículos esquerdo e direito. O objetivo deste estudo foi avaliar a deformação (St e StR) miocárdica radial do VE em modelo experimental de suínos com HAP induzida e tratados com angiotensina-(1-7), a fim de verificar as possibilidades desse novo fármaco nas respostas clínica e hemodinâmica, pois apresenta efeitos anti-inflamatório e vasodilatador, bem como ações antiproliferativas no sistema cardiovascular. Neste estudo, foi possível observar que os animais tratados com Ang-(1-7) apresentaram St e StR radiais maiores que o grupo placebo aos 60 dias de experimento, demonstrando uma melhora na função sistólica do miocárdio pelo aumento da deformação

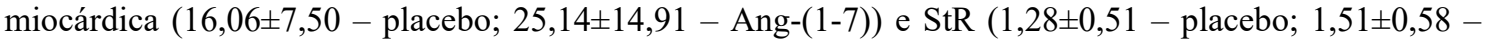
Ang-(1-7)). Essa melhora na função sistólica pode ser atribuída aos efeitos do fármaco, que reduziram também o desenvolvimento da hipertensão pulmonar. Diante dos resultados, acredita-se que a Ang-(1-7) possa ser um medicamento promissor para tratamento da HAP.

Palavras-chave: suíno, angiotensina-(1-7), strain radial, strain rate

\begin{abstract}
Pulmonary arterial hypertension (PAH) is a complex disease characterized by endothelial dysfunction resulting in pulmonary vascular remodeling, increased pulmonary arterial pressure, with subsequent right heart failure. The two-dimensional speckle tracking (2D-STE) is one of the newer tools used for early assessment of ventricular function. The aim of this study was to evaluate the radial myocardial deformation (St and StR) of LV in experimental pigs with induced PAH and treated with Ang- (1-7), checking the possibilities of this new drug in clinical and hemodynamic response, since it has antiinflammatory effects and antiproliferative actions on the cardiovascular system. In this study we observed that animals treated with Ang (1-7) had radial St and StR higher than the placebo group at 60 days of the experiment demonstrating an improvement in systolic function of the myocardium by increased myocardial deformation (16.06 \pm 7.50 - placebo; $25.14 \pm 14.91$ - Ang (1-7)) and StR (1.28 \pm 0.51 - placebo; $1.51 \pm 0.58$ - Ang (1-7)). This improvement in systolic function can be attributed to drug effects while also reducing the development of pulmonary hypertension. Based on the results it is believed that Ang (1-7) may be a promising drug for the treatment of $\mathrm{PAH}$.
\end{abstract}

Keywords: pigs, Angiotensin-(1-7), radialstrain, strain rate

Recebido em 9 de fevereiro de 2015

Aceito em 11 de dezembro de 2015

E-mail: marianacoelhorc@gmail.com 


\section{INTRODUÇÃO}

Hipertensão arterial pulmonar (HAP) é definida por pressão média da artéria pulmonar maior que $20 \mathrm{mmHg}$ em repouso ou maior que $30 \mathrm{mmHg}$ durante o exercício (Serres et al., 2007) e caracterizada por um aumento progressivo da resistência vascular pulmonar (Archeret al., 2010). Acredita-se que a disfunção endotelial seja o primeiro passo na remodelação vascular das artérias pulmonares durante o desenvolvimento de HAP (Nicod, 2007), que culmina em uma produção reduzida de vasodilatadores, tais como óxido nítrico (NO) e a prostaciclina, e superprodução de vasoconstritores, tais como tromboxane A2 (Hoeper et al., 2002) e endotelina-1 (Brás-Silva e Leite-Moreira, 2008). O sistema reninaangiotensina (SRA) é muito mais complexo do que inicialmente previsto, possuindo outros peptídeos biologicamente ativos, como a angiotensina 1-7 (Ang-(1-7)). Nos últimos anos, novos componentes do SRA foram descobertos, como a enzima conversora de angiotensina 2 (ECA2) (Lazartigues et al., 2007), que favorece a degradação da angiotensina 2 em Ang-(1-7). A Ang-(1-7) é um heptapeptídeo que atua como anti-inflamatório, vasodilatador, com ações antiproliferativas no sistema cardiovascular, as quais se opõem aos efeitos prejudiciais da angiotensina 2, por meio do seu receptor específico (Li et al., 2013).

A indução da HAP por crotalina (CT) tem sido utilizada em estudos experimentais com ratos. A MCT é uma substância alcaloide, sintetizada quimicamente e encontrada em folhas e sementes da planta Crotalaria spectabilis (Torres et al., 1997). Sua forma ativa, crotalina pirrole (CTP), após uma injeção subcutânea única, pode causar lesão vascular no endotélio de vasos pulmonares, ativação plaquetária, reatividade pulmonar aumentada por vasoconstrição e hipertrofia da camada muscular média dos vasos pulmonares (Sawamura et al., 2009).

O speckle tracking bidimensional (2D-STE) é uma das mais recentes ferramentas da ecocardiografia que permite o acesso à função miocárdica ventricular de forma mais precoce (Chetboul, 2010). Estudos como de Nishimura et al. (2012) avaliaram a relação entre a deformação radial e a hipertrofia ventricular esquerda em pacientes hipertensos.
Assim, o objetivo deste estudo foi avaliar a deformação miocárdica radial do VE (strain e strain rate) em modelo experimental de suínos com HAP induzida e tratados com Ang-(1-7), a fim de verificar as possibilidades desse novo fármaco nas respostas clínica e hemodinâmica. Além disso, por meio desse modelo, objetiva-se estudar formas que permitam o diagnóstico precoce das alterações induzidas pela HAP, associando a isso os achados clínicos, os exames ecocardiográficos e anatomopatológicos.

\section{MATERIAL E MÉTODO}

O experimento foi realizado em 14 suínos da linhagem comercial TOPIGS (Landrace $\mathrm{x}$ Large White), no Centro Experimental de Suínos (CES) da instituição. Os animais foram desmamados aos 28 dias e permaneceram 40 dias na unidade de creche e 50 dias na unidade de crescimento, em baias individuais com comedouro semiautomático e bebedouro tipo chupeta, recebendo dieta padrão e água ad libidum. Os suínos utilizados eram saudáveis, sem sinais de alterações cardiovasculares ou pulmonares ao exame físico e ao exame ecocardiográfico. Todo o protocolo experimental foi realizado de acordo com as normas para o uso de animais experimentais da instituição, registrado sob o $n^{\circ}$ $002 / 2010$.

Para realização dos exames eletrocardiográficos (ECG) e ecocardiográficos, os animais foram sedados com diazepam $(0,5-1,0 \mathrm{mg} / \mathrm{kg})$ e azaperone $(4,0 \mathrm{mg} / \mathrm{kg})$, via intramuscular profunda, aguardando um período de repouso de 15 minutos. Após esse período, aos 40 dias de vida (T0), todos os animais receberam uma dose única de $15 \mathrm{mg} / \mathrm{kg}$ de Crotalina Sigma ${ }^{\circledR}$, administrada por via intravenosa, sendo divididos em dois grupos aleatórios e de forma cega de sete animais cada. O grupo 1 recebeu tratamento com Ang-(1-7) $(30 \mathrm{mcg} / \mathrm{kg} / \mathrm{dia}$, por 60 dias), e o grupo 2 recebeu tratamento placebo. Novos exames de ECG e ecocardiográficos foram realizados no dia 30 (T30) e no dia 60 (T60) após a aplicação de crotalina.

Para avaliação eletrocardiográfica, os animais foram posicionados em decúbito lateral direito, utilizando-se um aparelho de eletrocardiografia portátil. Foram medidos a frequência cardíaca, o ritmo, as ondas e os intervalos. O ECG foi registrado em $50 \mathrm{~mm} / \mathrm{s}$ e $\mathrm{N}$. 
Para os estudos ecocardiográficos, foi utilizado um aparelho de ecocardiografia (MyLab 40 Esaote $^{\circledR}$, Itália), com transdutores de varredura setorial eletrônica multifrequencial de $4-10 \mathrm{~Hz}$ e com acompanhamento eletrocardiográfico no monitor. Todos os exames foram realizados pelo mesmo ecocardiografista (RAL Muzzi), e os animais sedados contidos gentilmente em decúbito lateral direito e esquerdo. Avaliações ecocardiográficas convencionais foram obtidas pelos cortes paraesternais longitudinais direito e esquerdo. Todas as valvas foram examinadas pelo Doppler em cores, e suas velocidades foram medidas usando-se Doppler pulsado. A obtenção das imagens e as avaliações da ecocardiografia convencional foram realizadas como recomendado por Boon (2011) e Thomas et al. (1993).

Para a ecocardiografia bidimensional 2D-STE, três a cinco ciclos cardíacos consecutivos utilizando uma monitorização contínua do ECG foram armazenados em formato digital para análise off-line utilizando o software XStrain, algoritmo optical flow (versão 10.1, Esaote). Para o strain radial, cine-loops foram adquiridos a partir do corte paraesternal direito transversal, com taxas de frames médios de 63,10 frames/s, e seis medidas foram obtidas em duas imagens diferentes (três para cada imagem) no final da sístole para todas as variáveis estudadas, e, em seguida, a média foi obtida. Para $o$ acompanhamento do miocárdio, a borda do endocárdio foi marcada manualmente, e, logo após, marcou-se a borda epicárdica automaticamente pelo software. Vetores de velocidade foram apresentados na região avaliada, e, quando necessário, foi realizado ajuste manual das bordas da parede miocárdica. Gráficos e curvas das variáveis estudadas foram apresentados pelo software, e os valores de pico sistólico de St radial e StR radial foram mostrados para as regiões endocárdica e epicárdica (Tab. 1 e 2).

Para as avaliações morfométricas, o coração, o pulmão direito e o pulmão esquerdo foram pesados separadamente. Para a avaliação histológica, amostras do átrio direito, ventrículo direito, átrio esquerdo, ventrículo esquerdo e pulmão (lobos cranial direito, médio, caudal direito na região proximal e distal, cranial esquerdo, caudal esquerdo na região proximal e distal e o lobo acessório) foram fixadas em formol a $10 \%$, tamponado com fosfatos, no $\mathrm{pH}$ 7,2, 0,01M. Após a fixação, o material foi clivado, incluído em parafina, cortado a cinco micrômetros e corado pela técnica de hematoxilina e eosina (HE). No ventrículo esquerdo, as amostras foram coletadas da parte média do ventrículo, entre o ápice e a base. Os cortes também foram submetidos à coloração histoquímica pelo tricrômico de Masson para melhor visualização das fibras colágenas.

Para identificar células ativas no ciclo celular, foi realizada a marcação imuno-histoquímica (IHQ) com o anticorpo monoclonal anti-PCNA (antígeno nuclear de células em proliferação Dako, USA) na diluição de 1:2000, e o kit comercial Dual Link HRP (Dako, USA). A reativação antigênica foi realizada mediante irradiação em forno de micro-ondas, na potência máxima, por seis minutos, com as lâminas imersas em tampão Tris-EDTA pH 9,0. Como cromógeno, foi utilizado o DAB (3,3'diaminobenzidina) (Dako, USA).

Em relação à análise estatística, todos os dados foram testados quanto à normalidade utilizandose o teste Kolmogorov-Smirnov $(\mathrm{P}>0,05)$, e foi avaliada a homocedasticidade das variâncias empregando-se o teste Levene $(\mathrm{P}>0,05)$. As análises estatísticas foram realizadas mediante $\mathrm{O}$ uso do pacote estatístico IBM ${ }^{\circledR}$ SPSS para Windows, versão 20.0 (IBM ${ }^{\circledR}$, SPSS 2011).

As variáveis apresentaram distribuição normal (St e StR radial miocárdicos) e foram submetidas à análise de variância (ANAVA); quando significativas, as médias entre os tratamentos foram comparadas pelo teste $\mathrm{F}$ e, entre os tempos (dias), foram comparadas pelo teste de Tukey.

\section{RESULTADOS}

A eletrocardiografia foi obtida nos 15 suínos do estudo, e os principais resultados observados foram os seguintes: para o dia zero, os valores médios e os desvios-padrão dos parâmetros eletrocardiográficos analisados foram frequência cardíaca (bpm) de 128,71 17,75 ; duração da onda $\mathrm{P}$ de $0,046 \pm 0,005$ segundos e amplitude de $0,115 \pm 0,029 \mathrm{mV}$; intervalo PR de $0,08 \pm 0,01$ segundos; duração do complexo QRS de 0,052 $\pm 0,008$ segundos e amplitude de $0,429 \pm 0,190 \mathrm{mV}$; onda $\mathrm{T}$ de $0,14 \pm 0,08 \mathrm{mV}$; intervalo QT de $0,28 \pm 0,03$ segundos e eixo de 
$59,642 \pm 46,956^{\circ}$. No dia 60 , observou-se um aumento numérico na amplitude da onda $\mathrm{T}$, no intervalo QT e eixo cardíaco com deslocamento para a esquerda nos animais do grupo placebo em comparação com o grupo tratado.

O speckle tracking bidimensional mostrou ser uma ferramenta útil em suínos utilizando-se a visão paraesternal direita transversal para medir as variáveis do strain radial.

$\mathrm{Na}$ Tab. 1, pode-se observar que, para o grupo placebo, quanto aos dias de tratamento, foi observada diferença apenas aos 30 dias e maior deformação em relação ao dia 60. Já o grupo tratado com Ang-(1-7) apresentou diferença apenas aos 60 dias de tratamento, e a variável St se mostrou aumentada em relação aos outros dias avaliados. Ao se realizar a comparação dos grupos entre os dias de tratamento, foi observado que, para o dia zero, não houve diferença $(\mathrm{P}>0,05)$ entre os grupos, fato já esperado, uma vez que os animais possuíam a mesma idade e as mesmas características fisiológicas. Em contrapartida, aos 60 dias de tratamento, houve um aumento para o St do grupo Ang-(1-7) em relação ao grupo placebo.

Tabela 1. Deformação miocárdica (strain - \%) radial avaliada nos diferentes grupos (placebo $\times$ Ang-(1-

7)) e dias de tratamento (zero, 30 e 60 dias) dos suínos utilizados no estudo $(n=14)$

\begin{tabular}{|c|c|c|c|c|c|c|c|}
\hline \multirow{2}{*}{$\begin{array}{c}\text { Grupo } \\
(\mathrm{G})\end{array}$} & \multicolumn{3}{|c|}{ Dias de tratamento (DT) } & \multirow[t]{2}{*}{ Média } & \multicolumn{3}{|c|}{ Valor de P } \\
\hline & 0 & 30 & 60 & & G & DT & $\mathrm{G} \times \mathrm{DT}$ \\
\hline Placebo & $18,31 \pm 14,45 b$ & $25,44 \pm 21,15 \mathrm{aA}$ & $16,06 \pm 7,50 \mathrm{bB}$ & $20,19 \pm 16,71$ & 0,820 & 0,076 & $<0,001$ \\
\hline Ang-(1-7) & $17,94 \pm 12,81 \mathrm{~b}$ & $16,00 \pm 7,50 \mathrm{bB}$ & $25,14 \pm 14,91 \mathrm{aA}$ & $19,70 \pm 12,74$ & & & \\
\hline Média & $18,12 \pm 13,62$ & $20,72 \pm 16,52$ & $21,03 \pm 13,82$ & & & & \\
\hline
\end{tabular}

Médias seguidas por diferentes letras maiúsculas na coluna diferem entre si pelo teste $\mathrm{F}(\mathrm{P}<0,05)$.

Médias seguidas por diferentes letras minúsculas na linha diferem entre si pelo teste Tukey $(\mathrm{P}<0,05)$.

Na Tab. 2, têm-se os dados obtidos para a variável strain rate (StR). Pode-se observar que, para o grupo placebo, houve uma redução significativa da taxa de deformação. Quanto ao grupo tratado com Ang-(1-7), os valores obtidos aos 60 dias de tratamento não diferiram $(\mathrm{P}>0,05)$ do dia zero, e aos 30 dias houve redução do StR em comparação com os demais tempos. Ao se compararem os grupos em relação aos tempos de tratamento, assim como para o St, não houve diferença $(\mathrm{P}>0,05)$ entre os grupos quanto ao dia zero; para o dia 60, foi verificada diferença com aumento do StR no grupo tratado com Ang-(1-7).

Tabela 2. Taxa de deformação miocárdica (strain rate $-1 / s$ ) radial avaliada nos diferentes grupos (placebo $\times$ Ang-(1-7)) e dias de tratamento (zero, 30 e 60 dias) dos suínos utilizados no estudo $(\mathrm{n}=14)$

\begin{tabular}{|c|c|c|c|c|c|c|c|}
\hline \multirow{2}{*}{$\begin{array}{c}\text { Grupo } \\
\text { (G) }\end{array}$} & \multicolumn{3}{|c|}{ Dias de tratamento (DT) } & \multirow[t]{2}{*}{ Média } & \multicolumn{3}{|c|}{ Valor de $\mathrm{P}$} \\
\hline & 0 & 30 & 60 & & $\mathrm{G}$ & DT & $\mathrm{G} \times \mathrm{DT}$ \\
\hline Placebo & $1,52 \pm 0,65 \mathrm{a}$ & $1,69 \pm 0,98 \mathrm{aA}$ & $1,28 \pm 0,51 \mathrm{bB}$ & $1,51 \pm 0,77$ & 0,877 & 0,032 & $<0,001$ \\
\hline Ang-(1-7) & $1,60 \pm 0,79 \mathrm{a}$ & $1,36 \pm 0,64 \mathrm{bB}$ & $1,51 \pm 0,58 \mathrm{aA}$ & $1,49 \pm 0,68$ & & & \\
\hline Média & $1,56 \pm 0,73 a$ & $1,53 \pm 0,84 \mathrm{a}$ & $1,41 \pm 0,56 b$ & & & & \\
\hline
\end{tabular}

Médias seguidas por diferentes letras maiúsculas na coluna diferem entre si pelo teste $\mathrm{F}(\mathrm{P}<0,05)$

Médias seguidas por diferentes letras minúsculas na linha diferem entre si pelo teste Tukey $(\mathrm{P}<0,05)$.

Não houve diferenças entre o peso do coração e dos pulmões direito e esquerdo entre os animais tratados e placebo.

Em relação ao coração, microscopicamente, dois dos sete animais tratados apresentaram lesões discretas de megalocitose dos cardiomiócitos e espessamento de fibras cardíacas, e apenas em um deles havia um foco de necrose de miofibrila associado a infiltrado mononuclear discreto. $\mathrm{Na}$ coloração de tricrômico de Masson, não houve diferença em relação aos grupos, e na imunohistoquímica ocorreu marcação em um número moderado de núcleo dos cardiomiócitos de apenas um animal do grupo placebo.

As alterações pulmonares foram encontradas de forma aleatória em diferentes regiões dos lobos pulmonares. Áreas com discreto a moderado espessamento de septo por congestão e material 
eosinofílico (fibrina) no interstício foram observados nos animais tratados. Já nos animais do grupo placebo, foi possível observar áreas com moderado a acentuado espessamento com material eosinofílico (necrose fibrinoide) e infiltrado inflamatório perivascular mononuclear moderado, artérias e arteríolas com moderado aumento de volume de células endoteliais que se projetam para a luz do vaso e moderada vacuolização nuclear das células da camada média, assim como vasculite, principalmente na camada adventícia, levando à dissociação das fibras. Além disso, observou-se imunohistoquímica com marcação positiva em células das camadas média e endotelial, e a coloração de tricrômico de Masson demonstrou a descontinuidade da camada média, bem como seu espessamento devido ao aumento do número de fibras colágenas nessa camada.

\section{DISCUSSÃO}

Neste estudo e no trabalho de Chen et al. (2011), a Ang-(1-7) mostrou ser efetiva no tratamento da HAP induzida por crotalina, assim como na remodelação vascular da hipertensão pulmonar. Seu mecanismo parece estar associado com a regulação positiva da ativação do óxido nítrico (NO) via receptores "shear" nas células endoteliais (Brunneret al., 2006).

A Ang-(1-7) tem amplos efeitos no sistema cardiovascular, incluindo a vasodilatação, a proteção miocárdica, bem como os efeitos antiarrítmico e anti-hipertensivo, inotrópico positivo e a inibição da remodelação cardíaca patológica (Santos et al., 2013). Os efeitos benéficos da Ang-(1-7) na hipertensão não são apenas relacionados ao efeito hipotensor, mas também à redução na lesão de órgão-alvo. Grobe e colaboradores (2006) descobriram que a infusão crônica de Ang-(1-7) impediu a deposição de colágeno no modelo de hipertensão em ratos sem afetar a pressão arterial e a hipertrofia cardíaca. Em outro estudo de Benter et al. (2006), demonstrou-se que a Ang-(1-7) melhorou as funções renal, cardíaca e vascular em ratos espontaneamente hipertensos; dessa forma, pode-se inferir que o mesmo fato tenha ocorrido nos suínos do presente estudo.

Em relação ao ECG, o grupo tratado com Ang(1-7) não apresentou alterações em ondas e intervalos, porém o grupo placebo apresentou um aumento numérico na amplitude da onda $\mathrm{T}$, no intervalo QT e eixo cardíaco com deslocamento para a esquerda. Esses achados podem sugerir uma lesão miocárdica induzida pela crotalina.

Sobre a ecocardiografia 2D STE, o grupo tratado com Ang-(1-7) apresentou St e StR radiais maiores que o grupo placebo no dia 60. Essa melhora na função do miocárdio pode ser atribuída aos efeitos do fármaco, reduzindo também o desenvolvimento da hipertensão pulmonar e alterações na função miocárdica. Pei et al. (2010) demonstraram no estudo com ratos que a Ang-(1-7) retarda a hipertrofia cardíaca associada com a redução de deposição de colágeno no miocárdio em ratos espontaneamente hipertensivos. Dessa maneira, a contratilidade miocárdica se torna mais eficiente, o que é comprovado pelo aumento da deformação miocárdica. Isso parece ter acontecido também neste estudo em suínos.

Em contrapartida, o grupo placebo apresentou menor StR quando comparado ao grupo tratado. Esse fato pode ser devido ao agravamento da doença e do quadro clínico desses animais, o qual poderia estar afetando a função miocárdica do VE em consequência do aumento na quantidade de deposição de colágeno, com um incremento correspondente na largura $\mathrm{e}$ continuidade dos componentes fibrilares da matriz extracelular, que pode influenciar o espessamento da parede ventricular (Kouzu et al., 2011).

Assim como no estudo de Nishimura et al. (2012), no presente trabalho observou-se deformação radial reduzida em indivíduos com HAP (grupo não tratado). Uma possível explicação é o fato de que, em razão do remodelamento ventricular, há uma mudança na geometria das fibras miocárdicas e no mecanismo de torção delas, atenuando o aumento compensatório do $\mathrm{St}$ radial em indivíduos hipertensos, como demonstrado por Kouzu et al. (2011).

O espessamento das artérias ocorreu de forma aleatória nos lobos pulmonares no estudo de Pietra et al. (2004), sendo observados, num mesmo animal, vasos afetados e não afetados. Esse fato também ocorreu no presente estudo. Porém, pela análise histopatológica, o grau de vasos afetados aparenta ser maior nos animais não tratados, afetando mais acentuadamente 
determinados animais desse grupo. As principais lesões encontradas foram aumento de volume de células endotelial e da camada média, que, pela imuno-histoquímica, sugere que além da hipertrofia, essa célula realiza tentativa de se dividir, comprovando a hipertensão pulmonar.

Na coloração tricrômico de Masson, em alguns animais do grupo placebo, foi possível observar um aumento de tecido conjuntivo na camada média, sugerindo que o espessamento do vaso ocorre tanto pela deposição de tecido conjuntivo como pela hipertrofia das células musculares, sejam elas em divisão ou na tentativa de divisão de toda a camada do vaso, principalmente média e endotelial, uma vez que algumas células foram marcadas pela IHQ.

Algumas limitações devem ser apontadas. O número de animais, assim como o fato de ser uma doença induzida, pode trazer resultados diferentes se tivessem sido utilizados um maior $\mathrm{n}$ e animais naturalmente portadores de HAP. No entanto, a HAP ocorre raramente em suínos, e o emprego de um número maior de animais não é ideal, por questões éticas.

Outro fator limitante foi a utilização de anestesia para a realização dos exames ecocardiográficos e eletrocardiográficos. Como os suínos são animais inquietos e muito estressados, a não utilização de fármacos sedativos poderia implicar problemas de manejo e na resposta experimental, optandose, dessa forma, por utilizar o sedativo.

\section{CONCLUSÃO}

O estudo demonstrou um efeito positivo da Ang-(1-7) no modelo experimental de hipertensão pulmonar induzida em suínos, tanto no coração como nos pulmões. Os animais tratados tiveram uma melhora na função cardíaca em decorrência dos efeitos anti-inflamatório, vasodilatador e das ações antiproliferativas no sistema cardiovascular, diagnosticada pela ecocardiografia speckle tracing bidimensional e comprovada pelas avaliações histológicas e imuno-histoquímicas.

Ainda são necessários, no entanto, mais estudos para definir melhor os mecanismos de atuação da Ang-(1-7) no organismo, embora os resultados do presente estudo se mostrem promissores.

\section{AGRADECIMENTOS}

Ao professor Robson Augusto Souza dos Santos, da Universidade Federal de Minas Gerais ICB/UFMG, pela colaboração no desenvolvimento deste trabalho, à Coordenação de Aperfeiçoamento de Pessoal de Nível Superior (Capes) e à Fundação de Amparo à Pesquisa do Estado de Minas Gerais (Fapemig), pelo financiamento deste estudo.

\section{REFERÊNCIAS}

ARCHER, S.L.; WEIR, E.K.; WILKINS, M.R. Basic science of pulmonary arterial hypertension for clinicians: new concepts and experimental therapies. Circulation, v.121, p.2045-2066, 2010.

BENTER, I.F.; YOUSIF, M.H.; ANIM, J.T. et al. Angiotensin-(1-7) prevents development of severe hypertension and end-organ damage in spontaneously hypertensive rats treated with LNAME. Am. J. Physio. Heart C., v.290, p.684691, 2006.

BOON, J.A. Evaluation of size, function, and hemodynamics. In: Boon, J.A. (Ed.), Veterinary Echocardiography. 2nd ed. Wiley-Blackwell, Iowa, IA, USA, p. 206- 334.

BRUNNER, F.; SILVA, C.B.; CERDEIRA, A.S.; LEITE-MOREIRA, A.F. Cardiovascular endothelins: essential regulators of cardiovascular homeostasis. Pharmacol. Ther., v.111, p.508-531, 2006.

CHEN, L.; XIAO, J.; LI, Y.; MA, H. Ang-(1-7) might prevent the development of monocrotaline induced pulmonary arterial hypertension in rats. Eur. Rev. Med. Pharmacol. Sci., v.15, p.1-7, 2011.

CHETBOUL, V. Advanced techniques in echocardiography in small animals. Vet. Clin. North Am. Small Anim. Pract., v.40, p.529-543, 2010.

GROBE, J.L.; MECCA, A.P.; MAO, H.; KATOVICH, M.J. Chronic angiotensin-(1-7) prevents cardiac fibrosis in DOCA-salt model of hypertension. J. Physiol. Heart C., v.290, p.2417-2423, 2006.

HOEPER, M.M.; GALIE N.; SIMONNEAU, G.; RUBIN, L.J. New treatments for pulmonary arterial hypertension. Am. J. Resp. Crit. Care, v.165, p.1209-1216, 2002. 
KOUZU, H.; YUDA, S.; MURANAKA, A. et al. Left ventricular hypertrophy causes different changes in longitudinal, radial, and circumferential mechanics in patients with hypertension: a two-dimensional speckle tracking study. J. Am. Soc. Echocardiogr., v.24, p.192-199, 2011.

LAZARTIGUES, E.; LAVOIE, J.L. The Two fACEs of the Tissue Renin-Angiotensin Systems: Implications in Cardiovascular Diseases. Curr. Pharm. Des., v.13, p.1231-1245, 2007.

LI, G.; LIU, Y.; ZHU, Y. et al. ACE2 Activation confers endothelial protection and attenuates neointimal lesions in prevention of severe pulmonary arterial hypertension in rats. Lung, v.191, p.327-336, 2013.

NICOD, L.P. The endothelium and genetics in pulmonary arterial hypertension. Swiss. Med. Wkly., v.137, p.437-442, 2007.

NISHIMURA, K.; OKAYAMA, K.; INOUE, K. et al. Direct measurement of radial strain in the inner-half layer of the left ventricular wall in hypertensive patients. J. Vet. Cardiol., v.59, p.64-71, 2012.

PEI, Z.; MENG, R.; LI, G. et al. Angiotensin-(17) ameliorates myocardial remodeling and interstitial fibrosis in spontaneous hypertension: role of MMPs/TIMPs. Toxicol. Lett., v.199, p.173-181, 2010.

PIETRA, G.G.; CAPRON, F.; STEWART, S. et al. Pathologic Assessment of Vasculopathies in Pulmonary Hypertension. J. Am. Coll. Cardiol., v.43, p.25-32, 2004.
SANTOS, R.A.; FERREIRA, A.J.; VERANOBRAGA, T.; BADER, M.T. Angiotensinconverting enzyme 2, angiotensin-(1-7) and Mas: new players of the renin-angiotensin system. J. Endocrinol., v.216, p.1-17, 2013.

SAWAMURA, F.; KATO, M.; FUJITA, K. et al. Tadalafil, a long-acting inhibitor of PDE5, improves pulmonary hemodynamics and survival rate of monocrotaline-induced pulmonary artery hypertension in rats. J. Pharm. Sci., v.111, p.235-243, 2009.

SERRES, F.; CHETBOUL, V.; GOUNI, V. et al. Diagnostic value of echo-Doppler and tissue Doppler imaging in dogs with pulmonary arterial hypertension. J. Vet. Intern. Med., v.21, p.12801289, 2007.

SILVA, C.B.; MOREIRA, A.F.L. Efeitos miocárdicos da endotelina-1. Rev. Port. Cardiol., v.27, p.925-951, 2008.

THOMAS, W.P., GABER, C.E., Jacobs, G.J. et al. Recommendations for standards in transthoracic two-dimensional echocardiography in the dog and cat. Echocardiography Committee of the Specialty of Cardiology, American College of Veterinary Internal Medicine. J. Vet. Intern. Med., v.7, p.247-252.

TORRES, M.B.A.M.; SALLES, M.W.S.; HEADLEY, S.A.; BARROS, C.S.L. Intoxicação experimental por sementes de Crotalaria spectabilis (leguminosae) em suínos. Ciênc. Rural, v. 27, p. 307-312, 1997. 